\title{
Kasuistiken
}

Rechtsmedizin $2020 \cdot 30: 106-111$ https://doi.org/10.1007/s00194-020-00375-3 Online publiziert: 13. Februar 2020

(c) Der/die Autor(en) 2020

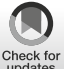

J. Schädler · A. Gehl · K. Püschel

Institut für Rechtsmedizin Hamburg, Universitätsklinikum Hamburg-Eppendorf, Hamburg, Deutschland

\section{Penetrierende Schädelverletzungen mit elektrischen Bohrmaschinen - ungewöhnliche Verletzungsmuster}

\section{Einführung}

Die Beurteilung von stumpfer Gewalt sowie von Stich- und Schussverletzungen gehört zur gängigen rechtsmedizinischen Praxis. Selbst- oder fremdbeigebrachte Schädelverletzungen durch Schusswaffen werden, z.B. bei Gewalteinwirkung gegen den Kopf, häufig beobachtet. Dahingegen sind penetrierende Schädelverletzungen, hervorgerufen durch ungewöhnliche (Stich-)Werkzeuge, wie Messer [4], Nagel, Schraubendreher [6, 16, 18], Axt [9], aber auch elektrische Werkzeugen, wie Kettensäge, Bohrmaschine oder Bolzenschussgeräte bzw. Geschosse mit einer Armbrust [1, 4, 11], selten. In den meisten Kasuistiken handelt es sich hierbei um einen (versuchten) Suizid von Männern im Alter zwischen 50 und 80 Jahren oder akzidentielle Verletzungen [11]. Einzelne Fallberichte beschreiben aber auch Tötungsdelikte mittels Schraubendreher $[6,7,16]$, Jagdmesser [4], Armbrust [4] oder Axt [9]. Bislang wurden in der Literatur Verletzungen durch elektrische Bohrmaschinen nur im Zusammenhang mit Suiziden oder Unfällen beschrieben $[1,8,14]$. Nachfolgend sollen ein versuchtes sowie ein vollendetes Tötungsdelikt durch elektrische Bohrmaschinen dargestellt und die entsprechenden Verletzungsmuster näher analysiert werden.

\section{Falldarstellung}

Fall 1

Der 47-jährige Geschädigte verbrachte einen gemeinsamen Abend mit seiner Adoptivtochter und seiner Exfrau (der späteren Täterin) in seiner Wohnung. Als der Mann eingeschlafen war, nahm seine Exfrau eine insgesamt $65-70 \mathrm{~cm}$ lange Bohrmaschine (- Abb. 1a) aus dem Abstellraum und rammte den Bohrer als Stichwerkzeug in das rechte Ohr ihres geschiedenen Mannes. Der im Bohrfutter eingespannte Bohrer hatte eine Länge von $35 \mathrm{~cm}$ und einen Durchmesser von $10 \mathrm{~mm}$. Anschließend floh die Ehefrau. Der Geschädigte konnte sich aus der Wohnung retten und machte im Flur des Mehrfamilienhauses auf sich aufmerksam, bevor er dort bewusstlos zusammenbrach. Anschließend wurde er in ein Krankenhaus gebracht und notoperiert. Er überlebte den Vorfall. Die Bohrmaschine wurde mit einer verbogenen Bohrerspitze vorgefunden. Ob der Bohrer bereits zuvor verbogen war oder durch das Zustechen verbogen wurde, blieb unklar.

Verletzungsmuster. Gemäß dem Operationsbericht verlief der Wundkanal ( Abb. 1b) vom äußeren Gehörgang in Richtung Schädelmitte mit geringer Neigung nach frontal. Nachdem der Bohrer in die Concha auricularis eingedrungen war und den knorpeligen Gehörgang zerfetzte, rutschte das Werkzeug am Mastoid ab und verlief entlang des auf- steigenden Unterkieferasts nach schräg vorn-unten. Der Stichkanal lag kranial und dorsal vom N. facialis, sodass dieser unverletzt blieb. Dahingegen kam es zur Verletzung der V. jugularis interna, Ruptur der A. occipitalis und einem Riss des N. accessorius in der Nähe des Foramen jugulare. Weiterhin erfolgte eine Zerstörung des N. hypoglossus, welcher nur noch in einzelnen Fasern intraoperativ nachweisbar war. Auch der zum Os hyoideum ziehende M. stylohyoideus wurde grob zerfetzt. Weiterhin wurde die Glandula parotis mit dem Bohraufsatz durchbohrt, wobei einzelne Äste des N. facialis intraglandulär zerstört wurden und $\mathrm{zu}$ einer peripheren Fazialisparese führten. Zusätzlich wurde bei der rechtsmedizinischen Untersuchung im Krankenhaus rechts oben am Brustkorb eine $10 \mathrm{~cm}$ lange von innenoben nach außen-unten absteigende geriffelte Hautabschürfung, die etwas unterhalb der rechten vorderen Achselfalte endete, festgestellt. Dies wurde als zweite Bohrereinwirkung interpretiert und entstand durch ein Abrutschen der Bohrmaschine bei einem ersten Versuch der Beschuldigten zuzustechen.

Letztlich führten die genannten Verletzungen neben einer Parese der betroffenen Nerven zu einem ischämischen Schlaganfall der rechten Großhirnhemisphäre im Stromgebiet der A. media cerebri. Das Opfer entwickelte postoperativ ein organisches Psychosyndrom mit Orientierungsstörung, psychomotorischer Verlangsamung und reaktiver 


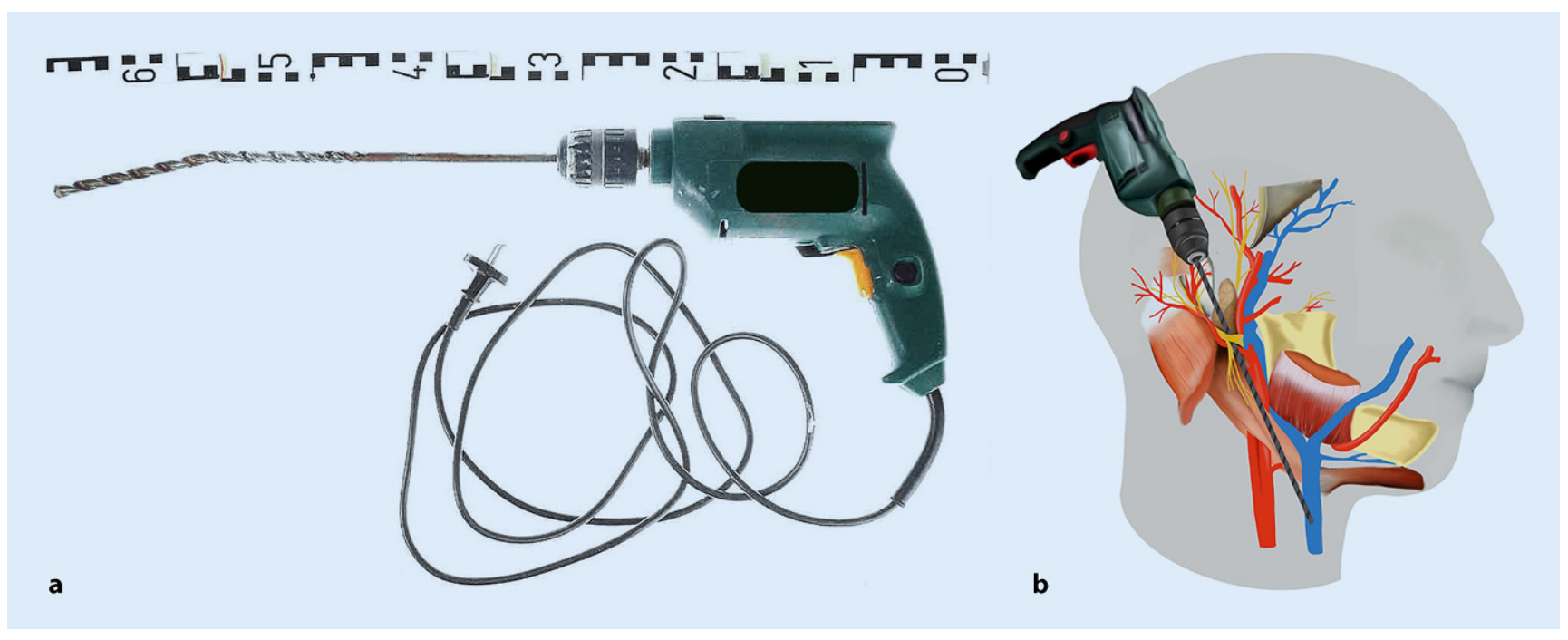

Abb. 1 ॥ Fall 1:a Tatwerkzeug, b Schemazeichnung des mutmaßlichen Stichkanals. (Zeichnung erstellt durch Paul Körner)

depressiver Verstimmung. Diese bildete sich im Verlauf der Krankenhaus- und Rehabilitationsbehandlung vollständig zurück. Eine armbetonte Hemiparese links mit geringer Spastik blieb nach abgeschlossener Behandlung bestehen.

Zunächst wurde ein Suizidversuch des Mannes sowohl durch die Polizei als auch aus rechtsmedizinischer Sicht erwogen. Nachdem der Geschädigte wieder ansprechbar war, bestritt er dies vehement und gab an, dass die Verletzungen durch seine Exfrau zugefügt wurden. Außerdem nahm er ein „Bohrgeräusch“ wahr. Nach Begutachtung des Tatwerkzeugs durch die Rechtsmedizin und Einsicht in die Ermittlungsakte konnte eine Selbstbeibringung in einer weiteren $\mathrm{Be}$ gutachtung ausgeschlossen werden. Bei einer Armlänge von Schulterhöhe zur Fingerspitze des Opfers von $70 \mathrm{~cm}$ und einer Gesamtlänge der Bohrmaschine von $65-70 \mathrm{~cm}$ schien eine Handhabung des Geräts zur Selbstbeibringung nahezu ausgeschlossen. Die wahrgenommenen Bohrgeräusche blieben letztlich unerklärlich: Die Beschuldigte gab in ihrer Einlassung an, mit dem Bohrer nur zugestochen zu haben. Es war am Tatort keine Steckdose verfügbar, die über das Stromkabel der Bohrmaschine erreicht werden könnte. Ein Verlängerungskabel wurde nicht in der Wohnung aufgefunden, und gemäß der Einlassung der Beschuldigten auch nicht verwendet. Hinsichtlich der verbogenen Bohrerspit- ze war davon auszugehen, dass diese entweder bereits vorher deformiert war oder nach dem Angriff verbogen worden ist. Eine Deformierung durch das Zustechen selbst wurde aufgrund der erfolgten Weichteilverletzungen ohne knöchernen Defekt und jedoch massiver Bohrerspitze mit $10 \mathrm{~mm}$ Durchmesser als wenig plausibel betrachtet.

Die beschuldigte Frau floh unmittelbar nach der Tat in ihr Herkunftsland. Sie wurde mittels internationalem Haftbefehl gesucht. Nachdem sie später festgesetzt wurde, erklärte sie ihre Beweggründe und gestand die Tat. Als Motiv nannte sie eine wiederholte sexuelle Gewalt durch ihren Exmann. Letztlich erhielt sie eine Haftstrafe von 3 Jahren und 4 Monaten.

\section{Fall 2}

Der 32-jährige Täter lernte im angetrunkenen Gemütszustand sein späteres 25jähriges, männliches Opfer in einer Bar kennen. Zusammen wurde weiter Alkohol konsumiert, wobei der Täter aus den Gläsern des Opfers trinken durfte. Zum Dank lud der 32-Jährige sein Opfer in die Wohnung seiner Freundin ein, um dort gemeinsam einen Joint zu rauchen. Aus Wut und Enttäuschung über sein Leben schlug die Stimmung des Täters plötzlich in eine allgemeine Aggressivität um. Als sein Opfer ihm den Rücken zuwandte, stach er mit einem großen Fleischer- messer (Gesamtlänge $34 \mathrm{~cm}$, Klingenlänge $21 \mathrm{~cm}$, Klingenbreite $5 \mathrm{~cm}$ ), welches er sich zuvor aus der Küche geholt hatte, als der andere Mann auf Toilette war, zehnmal auf sein Opfer ein. Nachdem dieses zu Boden sank, setzte der Täter weitere drei Stiche relativ parallel zueinander in den Hals. Drei weitere, oberflächliche Schnittverletzungen des Gesichtes des Opfers entstellten dieses hochgradig. Das Opfer gab zu diesem Zeitpunkt immer noch Lebenszeichen von sich. Entsprechend den Angaben des Täters "grinste" der bereits vielfach durch das Messer Verletzte weiterhin, als er rücklings auf dem Boden lag. Der Täter ergriff jetzt die elektrische Bohrmaschine, die noch von Handwerkstätigkeiten am Morgen im Zimmer auf dem Boden lag, und begann dem Geschädigten durch das linke Auge in den Schädel zu bohren (Auffindesituation: - Abb. 2a). Vorher schloss er das Gerät an den Strom an und montierte den Bohrer im Bohrfutter. Die Länge der Spiralbohrerspitze, gemessen ab dem Bohrfutter, betrug $19 \mathrm{~cm}$, der Durchmesser $15 \mathrm{~mm}$.

Verletzungsmuster. Bei der Sektion sowie bei der zuvor durchgeführten postmortalen Computertomographie (• Abb. 2b-d) wurden drei Bohrkanäle identifiziert, die zusammen durch die linke Orbita in die Schädelhöhle eindrangen. Nach Perforation des linken Augenunterlids folgte nach Durchtritt 
durch die Augenhöhle, ohne Verletzung des Augapfels, die grobe Zerstörung der Lamina papyracea des Os ethmoidale und der harten Hirnhaut in diesem Bereich mit Verlagerung von Knochensplittern bis weit in die Schädelhöhle und in die Hirnsubstanz hinein (- Abb. 2b, c). Durch wiederholtes Zurückziehen und Vorschieben des Bohrers entstanden letztlich drei separate, unterblutete Bohrkanäle (• Abb. 2d, e). Ein Bohrkanal endete nach Durchbruch des Os frontale in einem $2 \times 2 \mathrm{~cm}$ messenden knöchernen Defekt im hinteren Anteil der vorderen linksseitigen Schädelgrube. Die anderen beiden Wundkanäle führten $\mathrm{zu}$ einem $3 \times 2,5 \mathrm{~cm}$ messenden knöchernen Defekt der Schädelbasis: Sie durchbrachen den linksseitigen Anteil der Sella turcica mit nachfolgender Zertrümmerung des Orbitabodens, des Sinus frontalis, der Cellulae ethmoidale beidseits, des Sinus sphenoidalis, der Fossa hypophysialis sowie der Ala minor ossis sphenoidalis mit Einbezug eines Anteils der rechten Orbita. Letztlich endete ein Bohrkanal im vorderen Mittelhirnbereich im rechten Schläfenlappen. Der andere verlief entlang den rechten Basalganglien mittelliniennah bis nahe zum Einstrahlungsbereich der Pedunculi cerebri. Beide Wundkanäle betrugen $13-14 \mathrm{~cm}$. Durch die Gewalteinwirkung wurde auch die rechte Orbita bzw. der rechte Augapfel deformiert, obwohl hier kein eigener Bohrkanal vorlag. Die Bohrkanäle konnten in der postmortalen Computertomographie insbesondere über Gaseinschlüsse (• Abb. 2c) entlang den Verletzungen sowie bis in die Tiefe des Hirnparenchyms mitgerissene Knochenfragmente (- Abb. 2b, c) rekonstruiert werden.

Weiterhin konnten im Gesicht, beginnend im Zentrum der linken Wange ( Abb. 2f), eine $12 \mathrm{~cm}$ messende, bogenförmige, im Wundgrund geriffelte, bis $1 \mathrm{~cm}$ tiefe, bis teils auf den Knochen reichende Schnittverletzung sowie eine $4,5 \mathrm{~cm}$ lange, morphologisch ähnliche Verletzung in der Stirnregion festgestellt werden. Diese imponierten, als wären sie durch ein Abrutschen der Bohrmaschine entstanden.

Bereits die Messerstiche führten $\mathrm{zu}$ akut lebensbedrohlichen Verletzungen.

Rechtsmedizin 2020 · 30:106-111 https://doi.org/10.1007/s00194-020-00375-3

(c) Der/die Autor(en) 2020

\section{J. Schädler · A. Gehl · K. Püschel}

\section{Penetrierende Schädelverletzungen mit elektrischen Bohrmaschinen - ungewöhnliche Verletzungsmuster}

\section{Zusammenfassung}

Zwei außergewöhnliche Fälle von fremdbeigebrachten penetrierenden Kopfverletzungen durch elektrische Bohrmaschinen werden präsentiert. Derartige Abläufe stellen als Tötungsdelikt bzw. versuchtes Tötungsdelikt eine extreme Rarität dar. Wenige Beschreibungen betreffen Suizide, Suizidversuche oder unfallbedingte Verletzungen durch Bohrmaschinen. Im Fall eines 47 Jahre alten Mannes wurde dem Opfer von seiner in Scheidung lebenden Exfrau mit einer ausgeschalteten Bohrmaschine im Schlaf durch das Ohr in den Schädel gestochen. Der Mann überlebte den
Angriff. In dem anderen Fall kam es zu einer Auseinandersetzung zwischen zwei jungen Männern, wobei der Beschuldigte seinem Opfer zunächst Messerstiche und anschließend Bohrerverletzungen zufügte. Insgesamt konnten autoptisch drei durch das Auge in das Schädelinnere eindringende Bohrkanäle festgestellt werden. Das Opfer verstarb unmittelbar am Geschehensort.

\section{Schlüsselwörter}

Elektrische Bohrmaschine · Suizid · Tötungsdelikt · Penetrierende Schädelverletzungen . Computertomographie

\section{Penetrating head injury involving electric power drills—unusual injury pattern}

\begin{abstract}
This article presents two unusual cases of penetrating head injuries carried out by another person by means of electric power drills. Such procedures are an extreme rarity as homicidal or attempted homicidal attacks. Some descriptions involve suicides, suicide attempts or accidental injuries using electric power drills. In the case of a 47-year-old man, the victim was stabbed through the ear into the skull with a disconnected power drill while sleeping by his divorced ex-wife. The man survived the attack. The other
\end{abstract}

case involved an altercation between two young men, whereby first knife wounds and then drilling injuries were inflicted by the perpetrator. During the autopsy three drilling wound canals, which ran through the left eye into the skull could be determined. The victim died immediately at the scene of the crime.

\section{Keywords}

Electric power drill · Suicide · Homicide · Penetrating head injury $\cdot$ Computed tomography
Zum Zeitpunkt des Bohrens lebte das Opfer noch. Dies zeigte sich an den stark unterbluteten Bohrkanälen und deckt sich mit den Angaben des Beschuldigten. Die durch das Bohren gesetzten Verletzungen führten unmittelbar zum Tod.

Der Getötete wies bei der Sektion außerdem zahlreiche aktive und passive Abwehrverletzungen an den Armen und Händen auf. Todesursächlich war ein Verbluten, bedingt durch die zahlreichen Stichverletzungen in Kombination mit einer erheblichen Hirngewebszerstörung durch die massiven Bohrverletzungen.

Toxikologische Ergebnisse. Bei dem getöteten Mann konnten eine nicht unerhebliche Alkoholisierung mit einer
Blutalkoholkonzentration (Venenblut) von 1,6\% sowie ein Cannabiskonsum (Venenblut, $0,14 \mathrm{mg} / \mathrm{l}$ ) nachgewiesen werden, welches auf einen todeszeitnahen Konsum hinwies. Der Beschuldigte teilte mit, dass der Geschädigte kurz vor dem Angriff einen Joint rauchte. Der Beschuldigte war einen regelmäßigen Alkoholkonsum gewohnt und zeigte trotz einer Blutalkoholkonzentration von $1,92 \%$ keine offensichtlichen Ausfallserscheinungen. In den etwa 2 Stunden nach dem Tatzeitpunkt entnommenen Blutproben konnte zudem das unwirksame Abbauprodukt TetrahydrocannabiolCarbonsäure in niedriger Konzentration $(0,0061 \mathrm{mg} / \mathrm{l})$ nachgewiesen werden. Der Beschuldigte gab an, etwa 7 Stun- 

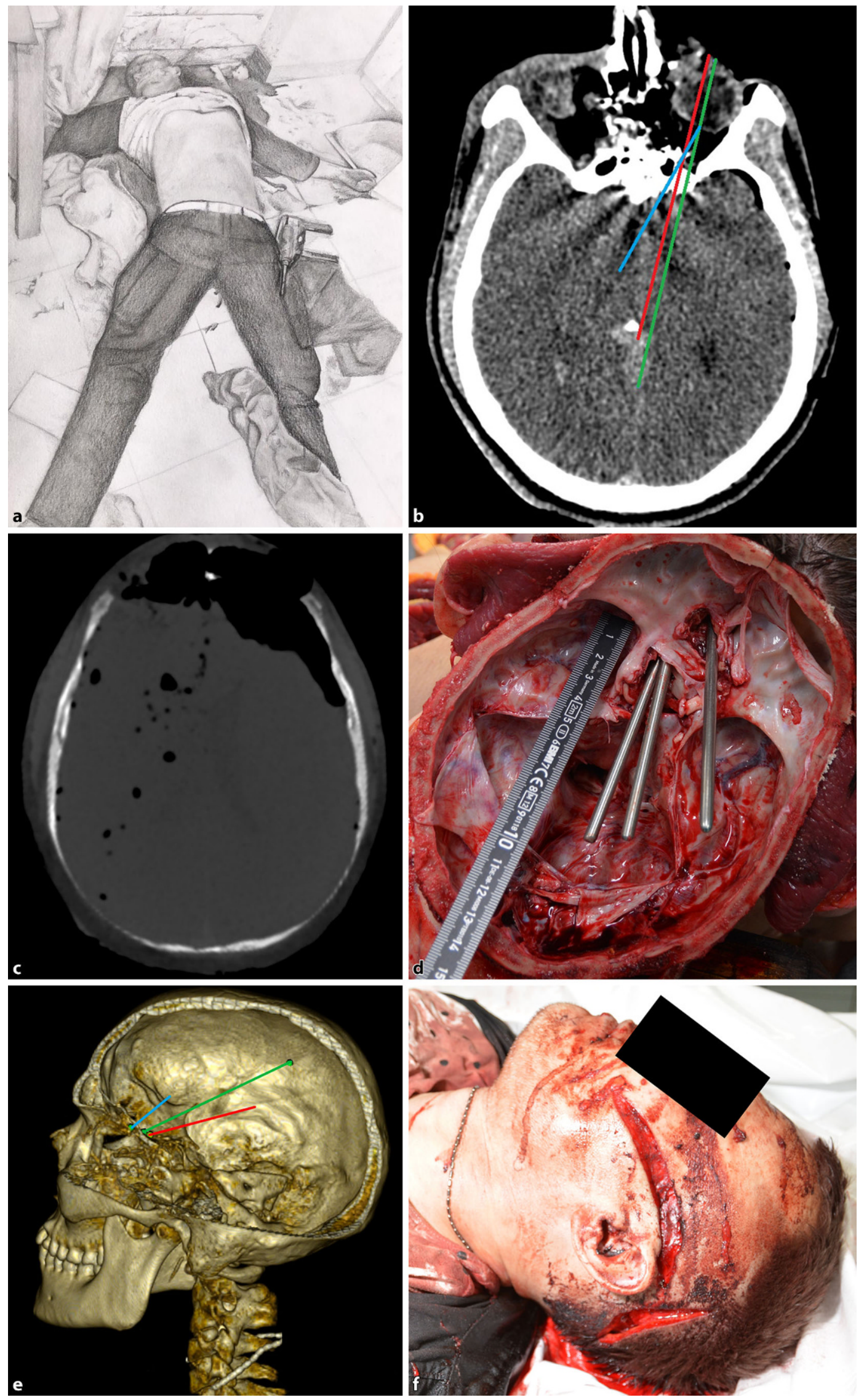

Abb. $2<$ Fall 2: a Zeichnerische Darstellung der Auffindesituation des Getöteten am Tatort. (Zeichnung erstellt durch Paul Körner). b Computertomographische Darstellung der Bohrkanäle. c Lufteinschlüsse im Verlauf der Bohrkanäle. d Rekonstruktion der Bohrkanäle. e Sondierung der Bohrkanäle während der Sektion.f Verletzung der linken Wange infolge eines Abrutschens mit der Bohrmaschine 
den vor der Tat einen Joint konsumiert $\mathrm{zu}$ haben. Alle Angaben hinsichtlich des Drogenkonsums deckten sich mit den Ergebnissen der toxikologischen Untersuchungen.

Letztlich wurde der Täter zu einer lebenslangen Haftstrafe verurteilt.

\section{Diskussion}

In der Literatur finden sich bis jetzt ausschließlich Fallberichte über Verletzungen, die durch Unfälle mit elektrischen Bohrmaschinen akzidentiell [5] oder in suizidaler Absicht verursacht worden sind. Dabei wurden die suizidalen Handlungen in allen Fällen durch Männer im Alter zwischen 25 und 80 Jahren mit bekannter Depression oder anderweitiger psychiatrischer Erkrankung durchgeführt. Frauen fanden sich keine unter den genannten Suizidenten $[2,5,8,10$, 14, 15, 17]. Bereits der Serienmörder Dahmer brachte vieren seiner 17 Opfer, die er in den Jahren 1978-1991 getötet hatte, noch während sie lebten, den Schädel penetrierende Bohrverletzungen bei [13]. Sein Ziel war es, entsprechend seinen Aussagen, sich „Zombie-SexSklaven“ durch die Injektion von Salzsäure oder andere Materialien in den Bohrkanal zu erschaffen [12, 13].

Aus rechtsmedizinischer Sicht ist bei Verletzungen durch elektrische Bohrmaschinen die Differenzierung zwischen fremdbeigebrachten Verletzungen und Verletzungen in suizidaler Absicht u. U. diffizil, wie das erste Fallbeispiel belegt. Als wichtige Aspekte einer Selbstbeibringung sollte neben dem Vorliegen von aktiven und passiven Abwehrverletzungen oder Probier- und Zauderverletzungen insbesondere die Erreichbarkeit des Eintrittsortes des Suizidwerkzeugs berücksichtigt werden. Im oben genannten Fall 1 ließ v.a. das Fehlen von Abwehrverletzungen den Verdacht eines Suizids aufkommen. Da der Mann jedoch im Schlaf angegriffen worden ist, hatte er keine Möglichkeiten, sich $\mathrm{zu}$ wehren. Die Verletzung am Oberkörper wurde zunächst als Zauderverletzung interpretiert, war letztlich aber durch ein Abrutschen der Bohrmaschine im Rahmen des Angriffs entstanden. Erst die differenzierte Begutachtung mit einem
Vergleich der Konfiguration der verwendeten Bohrmaschine (Gesamtlänge nahezu $70 \mathrm{~cm}$ ) und der Biometrie des Arms des Geschädigten schloss einen Suizid aus.

Aufgrund der schwerwiegenden Kopfverletzungen im Fall 1 hat das Bett bzw. die Matratze in gewisser Weise als Widerlager fungiert. Letztlich wurde nur Weichteilgewebe (Muskulatur, Nerven, Gefäße) verletzt. Für eine Penetration des massiven Schädelknochens, insbesondere des Mastoids, reichte die Krafteinwirkung bei ausgeschalteter Bohrmaschine nicht aus.

In der Literatur sind in allen Fällen mit suizidaler Absicht ausschließlich penetrierende Schädel-Hirn-Verletzungen, insbesondere der rechten Scheitel-Schläfen-Region [2, 5, 8, 14, 17] sowie Perforationen am Thorax genannt [10, 15, 17]. Die Verletzungsmuster gestalten sich dabei unterschiedlich: Die Wundkanäle im Kopfbereich verlaufen eher von unten nach oben und die im Thoraxbereich eher von oben-außen nach unten-innen [17]. Im Bereich des Schädels sind häufig mehrere Verletzungen beschrieben, wobei einige nur die Kopfschwarte und den Knochen im Sinne eines Bohrlochs verletzten, ohne den Schädel jedoch vollständig zu eröffnen [17]. Vereinzelt werden auch Zauderverletzungen beschrieben. Hausmann et al. beobachteten z. B. drei oberflächliche, punktförmige Hautmarken in der Nähe der Eintrittswunde und interpretierten diese als Zauder-bzw. Probierverletzungen [10].

Durchbricht die Bohrerspitze den Schädelknochen, kann sie bis tief in das weiche Hirngewebe eindringen [2]. Dabei kommt es, wie im Fall 2 beobachtet, zu einer ausgedehnten Gewebszerstörung mit Mitreißen von zahlreichen knöchernen Fragmenten bis in die Tiefe des Gehirns [17]. Im vorgestellten Fall 2 war ein Verbluten aufgrund der multiplen Stichverletzungen in Kombination mit der massiven Hirngewebszerstörung todesursächlich. In einigen Fällen, vorausgesetzt, dass kein größeres Gefäß oder ein Gehirnsinus durch den Bohrkanal getroffen wurde, waren die Geschädigten zunächst noch handlungsfähig. Kelly et al. beschreiben beispielsweise, dass der Patient zunächst ohne neurologische
Auffälligkeiten mit zwei Bohrverletzungen der Kopfhaut ohne knöcherne Verletzung ins Krankenhaus eingeliefert worden ist. Erst 11 Tage später wurden aufgrund einer Infektion zwei Bohrkanäle durch den Schädelknochen mit Hirnparenchymverletzungen festgestellt [14]. In einem anderen Fall war der Suizident noch in der Lage, nachdem er mit einem Bohrer eine penetrierende Schädelverletzung mit subarachnoidaler Blutung hervorgerufen hatte, sich vier weitere Stichverletzungen mit einem Gemüsemesser zuzufügen, welche schlussendlich zum Eintritt des Todes führten. Erst die Analyse der Blutspuren und das Fehlen von Abwehrverletzungen sowie die psychiatrische Erkrankung stärkten letztlich die Annahme eines Suizids [2].

Als charakteristische Wundmorphologie lässt sich beobachten, dass der durch die Bohrmaschine gesetzte annähernd kreisrunde Defekt der Kopfschwarte kleiner ist als der knöcherne Defekt beispielsweise im Schädelknochen [17]. Wird der Bohrer bis zum Bohrfutter eingeführt, kann um den Weichteildefekt eine kreisrunde Hautvertrocknungszone entstehen, die letztlich dem Abdruck der Mündung einer Schusswaffe bei einem aufgesetzten Schuss ähneln kann [10, 17]. Der Hautdefekt, verursacht durch die elektrische Bohrmaschine, kann somit möglicherweise als Einschussloch fehlinterpretiert werden. In den beiden oben beschriebenen Fällen konnten zudem geriffelte bzw. gewundene Oberhautabschürfungen nahe der Eintrittspforte des Bohrers feststellt werden. Im Fall 1 wurden diese zunächst als Zauderverletzungen interpretiert. Abschließend stellte sich jedoch raus, dass die Täterin in einem ersten Versuch mit der Bohrmaschine abgerutscht war. Auch im zweiten Fall ließ sich rekonstruieren, dass der Täter zunächst versuchte, den Schädel im Stirn- und im Wangenbereich $\mathrm{zu}$ eröffnen, jedoch abrutschte und sich schließlich für das Auge als Bohrlokalisation entschied.

Die Eintrittspforten in den beiden vorgestellten Fällen unterscheiden sich von denen in selbstbeigebrachten Fällen. Mit dem Ohr bzw. dem Auge haben beide Täter sich eine Struktur des Kopfes gesucht, die aus anatomischer Sicht auf- 
grund der dünnen knöchernen Wand vergleichsweise einfach mit einem Bohrer bzw. Werkzeug allgemein zu perforieren ist, um in das Schädelinnere einzudringen. Im zweiten Fall wurde sicherlich u.a. auch aufgrund des "Grinsens" des Opfers, welches der Täter wahrgenommen haben will, das Auge als Bohrpunkt gewählt. Bei akzidentiell zugefügten Verletzungen mit elektrischen Bohrmaschinen oder Schraubendrehern zeigte sich ebenfalls häufiger das Auge bzw. die Augenhöhle oder der Oberkiefer als Eintrittspforte für penetrierende, tiefreichende Schädel- bzw. Hirngewebsverletzungen $[3,5,18]$.

Gutiérrez-González et al. beschrieben einen Fall, in dem ein 80-jähriger depressiv erkrankter Mann sich mehrfach in den Schädel bohrte und letztlich die Bohrerspitze abbrach. Diese konnte erfolgreich operativ entfernt werden. Der Mann überlebte den Suizidversuch [8].

Zusammengefasst scheinen isolierte Verletzungen durch elektrische Bohrmaschinen im Schädelbereich eher seltener zum unmittelbaren Todeseintritt zu führen [8]. Penetrierende Bohrverletzungen des Schädels sind häufig erst gemeinsam mit anderen Verletzungen, wie z.B. die im Fall 2 beschriebenen Stichverletzungen, oder zusätzlichen Bohrkanälen, wie z. B. im Thorax mit Eröffnung des Herzbeutels, des Herzens und Zerstörung der Lunge, todesursächlich $[10,15,17]$.

\section{Fazit}

Elektrische Bohrmaschinen sind als Tatwerkzeug sowohl für suizidale Handlungen als auch zur Fremdbeibringung von Relevanz, da sie, im Gegensatz zu z. B. Schusswaffen, für jeden gut zugängig sind und in unterschiedlichen Größen in fast jedem Haushalt vorkommen. Eine sorgfältige rechtsmedizinische klinische Untersuchung bzw. Sektion mit Inspektion des mutmaßlichen Tatwerkzeugs ist von Bedeutung, um zwischen Selbstund Fremdbeibringung zu differenzieren. Auf die Blutspurenverteilung, Abwehrverletzungen sowie auf anhaftende Gewebsreste an der Bohrmaschine sollte geachtet werden. Differenzialdiagnostisch kann z.B. eine Schussverletzung bedacht werden. In den vorgestellten
Fällen eines vollendeten bzw. versuchten Tötungsdeliktes konnten zudem geriffelte bzw. gewundene oberflächliche Hautabschürfungen festgestellt werden, die von einem Abrutschen der Bohrmaschine stammten und nicht mit Zauderbzw. Probierverletzungen bei einem Suizid verwechselt werden sollten.

\section{Korrespondenzadresse}

\section{Dr. med. J. Schädler}

Institut für Rechtsmedizin Hamburg, Universitätsklinikum Hamburg-Eppendorf Butenfeld 34, 22529 Hamburg, Deutschland j.schaedler@uke.de

Funding. Open Access funding provided by Projekt DEAL.

\section{Einhaltung ethischer Richtlinien}

Interessenkonflikt. J.Schädler, A.Gehl und K. Püschel geben an, dass kein Interessenkonflikt besteht.

Für diesen Beitrag wurden von den Autoren keine Studien an Menschen oder Tieren durchgeführt. Für die aufgeführten Studien gelten die jeweils dort angegebenen ethischen Richtlinien. Für Bildmaterial oder anderweitige Angaben innerhalb des Manuskripts, über die Patienten zu identifizieren sind, liegt von ihnen und/oder ihren gesetzlichen Vertretern eine schriftliche Einwilligung vor. Die Untersuchungen erfolgten unter Einhaltung der Vorgaben der Zentralen Ethikkommission der Bundesärztekammer.

Open Access. Dieser Artikel wird unter der Creative Commons Namensnennung 4.0 International Lizenz veröffentlicht, welche die Nutzung, Vervielfältigung, Bearbeitung, Verbreitung und Wiedergabe in jeglichem Medium und Format erlaubt, sofern Sie den/die ursprünglichen Autor(en) und die Quelle ordnungsgemäß nennen, einen Link zur Creative Commons Lizenz beifügen und angeben, ob Änderungen vorgenommen wurden.

Die in diesem Artikel enthaltenen Bilder und sonstiges Drittmaterial unterliegen ebenfalls der genannten Creative Commons Lizenz, sofern sich aus der Abbildungslegende nichts anderes ergibt. Sofern das betreffende Material nicht unter der genannten Creative Commons Lizenz steht und die betreffende Handlung nicht nach gesetzlichen Vorschriften erlaubt ist, ist für die oben aufgeführten Weiterverwendungen des Materials die Einwilligung des jeweiligen Rechteinhabers einzuholen.

Weitere Details zur Lizenz entnehmen Sie bitte der Lizenzinformation auf http://creativecommons.org/ licenses/by/4.0/deed.de.

\section{Literatur}

1. Byard RW, Koszyca B, James R (1999) Crossbow suicide: mechanisms of injury and neuropathologic findings. Am J Forensic Med Pathol 20(4):347-353

2. Byard RW (2013) Suicide attempts involving power drills. J Forensic Leg Med 20(8):1032-1034. https:// doi.org/10.1016/j.jflm.2013.09.013

3. Chibbaro S, Tacconi L (2006) Orbito-cranial injuries caused by penetrating non-missile foreign bodies. Experience with eighteen patients. Acta Neurochir (Wien) 148(9):937-941. https://doi.org/10.1007/ s00701-006-0794-5 (Diskussion 941-942)

4. Clerici CA, Muccino E, Gentile G, Marchesi M, Veneroni L, Zoja R (2015) An unusual case of homicide with a crossbow and a hunting knife. Med Sci Law 55(2):86-89. https://doi.org/10.1177/ 0025802414537253

5. Costantinides F (1982) A fatal case of accidental cerebral injury due to power drill. Am J Forensic Med Pathol 3(3):241-243

6. Di Vella G, Grattagliano I, Romanelli MC, Duval JB, Catanesi R (2017) The tragic tale of a father and son: an unusual patricide. Clin Ter 168(3):e173-e177. https://doi.org/10.7417/T.2017.2000

7. Faller-Marquardt M, Pollak S (1996) Homicide with a screwdriver and simulation of a similar offence by self-infliction of injuries. J Clin Forensic Med 3(3):141-147

8. Gutiérrez-González R, Boto GR, Rivero-Garvía $M$, Pérez-Zamarrón A, Gómez G (2008) Penetrating brain injury by drill bit. Clin Neurol Neurosurg 110(2):207-210. https://doi.org/10.1016/j. clineuro.2007.09.014

9. Handlos P, Uvíra M, Dokoupil M, Marecová K (2019) Axe injury pattern in homicide. Forensic Sci Med Pathol 15(3):516-518. https://doi.org/10.1007/ s12024-019-00112-7

10. Hausmann R, Betz P (2002) Suicide by a power drill. Forensic Sci Int 129(3):191-193

11. Hessler C, Hamel W, Kluge S, Mayer U, Grzyska U, Westphal M, Püschel K (2012) Tödliche Armbrustverletzung im Jugendalter. Arch Kriminol 229(3-4):90-95

12. Jentzen JM (2017) Micro disasters: the case of serial killer Jeffrey Dahmer. Acad Forensic Pathol 7(3):444-452. https://doi.org/10.23907/2017.037

13. Jentzen JM, Palermo G, Johnson LT, Ho KC, Stormo KA, Teggatz J (1994) Destructive hostility: the Jeffrey Dahmer case. A psychiatric and forensic study of a serial killer. Am J Forensic Med Pathol 15(4):283-294

14. Kelly AJ, Pople I, Cummins BH (1992) Unusual craniocerebral penetrating injury by a power drill: case report. Surg Neurol 38(6):471-472. https:// doi.org/10.1016/0090-3019(92)90118-7

15. Monticelli F, Meyer HJ, Keller T (2006) Suicide by an electric power drill. Forensic Sci Med Pathol 2(4):273-276. https://doi.org/10.1385/FSMP:2:4: 273

16. Nadjem H, Pollak S (1993) Erscheinungsformen von Schraubendreherverletzungen. Arch Kriminol 192(1-2):27-36

17. Schmeling A, Lignitz E, Strauch H (2003) Kasuistische Beiträge zum Suizid mit elektrischer Bohrmaschine. Arch Kriminol 211(3-4):65-72

18. Smrkolj V, Balazic J, Princic J (1995) Intracranial injuries by a screwdriver. Forensic Sci Int 76(3):211-216 